\title{
Postharvest application of gum arabic and essential oils for controlling anthracnose and quality of banana and papaya during cold storage.
}

\begin{abstract}
Management of anthracnose caused by Colletotrichum spp. is the most important issue for the tropical fruit industry because of resulting financial losses. Antifungal effects of gum arabic (GA) (10\%), lemongrass oil (LG) (0.05\%), cinnamon oil (CM) (0.4\%), and their combinations were investigated in vitro and in vivo for controlling postharvest anthracnose of banana and papaya. LG at $0.05 \%$ and $0.4 \% \mathrm{CM}$ showed fungicidal effects against Colletotrichum musae and Colletotrichum gloeosporioides, causal organisms of banana and papaya anthracnose, respectively. GA alone did not show any fungicidal effects while the combination of $0.05 \% \mathrm{LG}$ and $0.4 \% \mathrm{CM}$ with Ten percent GA alone showed more fungicidal effects. However, potato dextrose agar (PDA) medium modified with $10 \%$ GA combined with $0.4 \% \mathrm{CM}$ showed the most promising results among all treatments against $\mathrm{C}$. musae and C. gloeosporioides in suppressing the mycelial growth $(73.4 \%)$ and $(70.0 \%)$ and spore germination inhibition (88\%) and (85\%), respectively. In vivo studies also revealed that $10 \%$ GA combined with $0.4 \% \mathrm{CM}$ was the optimal concentration in controlling decay $(80 \%)$ and (71\%), showing a synergistic effect in the reduction of C. musae and C. gloeosporioides, respectively, in artificially inoculated bananas and papayas. The results regarding quality evaluation also confirmed the efficacy of $10 \%$ GA combined with $0.4 \% \mathrm{CM}$ coatings since ripening was significantly delayed, in terms of percentage weight loss, fruit firmness, soluble solids concentration and titratable acidity. The results suggest the possibility of using $10 \%$ gum arabic combined with $0.4 \%$ cinnamon oil as a biofungicide for controlling postharvest anthracnose in major tropical fruit such as banana and papaya.
\end{abstract}

Keyword: Carica papaya; Cinnamon oil; Colletotrichum gloeosporioides; Edible coatings; Lemongrass oil; Musa acuminata; Postharvest quality. 